\title{
THE ERGODIC DECOMPOSITION OF CONSERVATIVE BAIRE MEASURES ${ }^{1}$
}

\author{
MICHAEL L. STURGEON
}

\begin{abstract}
Certain topological conditions on a Markov transition function are shown sufficient for an integral representation of conservative invariant Baire measures. The analysis incorporates the Choquet-Bishop-de Leeuw extension of the KreinMilman theorem.
\end{abstract}

1. Introduction. A Markov process is prescribed here as a quartet $(X, \mathscr{B}, p(x, B), m)$, the components respectively a given state space, $\sigma$-algebra of events, Markov transition function, and any $\sigma$-finite initial measure satisfying the condition that all $m$-null events are invariant or equivalently that on $\mathscr{B}: m(B)=0 \Rightarrow m\{x: p(x, B)>0\}=0$. The requirement on $m$ is the obverse of the usual notion of a nonsingular transition function and will be called presubinvariance, a sine-qua-non for measures either invariant or conservative with respect to a given transition function.

The conservativeness of $m$, for our purposes, is best defined following S. R. Foguel [4], as

$$
\begin{array}{rlrlrl}
X=C(m) \Leftrightarrow \text { on } \mathscr{B}: \sum_{1}^{\infty} p^{n}(x, B) & =\infty & & \text { on } \tilde{B} & \text { ae }(m), \\
& =0 & & \text { elsewhere }
\end{array}
$$

where $\widetilde{B}$ is a minimal superset for $B$ satisfying $p(x, \widetilde{B})=1_{\widetilde{B}}$ ae $(m)$. When $m$ is known to be conservative, $\widetilde{B}$ can be replaced by the $m$-equivalent set $\hat{B}=B \cup\left\{x: 0<\sum_{1}^{\infty} p^{n}(x, B)\right\}$.

The invariance of $m$ is defined using the operator $(\cdot) T$ on signed measures, whereby $m$ is invariant (subinvariant) iff on $\mathscr{B}$ :

$$
m T(B) \equiv \int p(x, B) d m(x)=(\leqq) m(B) .
$$

Received by the editors May 8, 1973 and, in revised form, August, 20, 1973.

AMS (MOS) subject classifications (1970). Primary 28A65, $60 \mathrm{~J} 99$.

Key words and phrases. Ergodic decomposition, integral representation, invariant measures, conservative measures, Choquet representation, Markov transition functions, recurrence, Baire measures.

${ }^{1}$ This research was supported by Grants ONR 2216-26 and NSF GP-8770 at the University of California, San Diego, and represents a portion of the author's doctoral dissertation written under the guidance of Professor Murray Rosenblatt.

(c) American Mathematical Soziety 1974 
For invariant conservative $m$, the $\sigma$-algebra of invariant events, $\Sigma(m)$, is composed of sets $B$ satisfying $\widetilde{T} 1_{B}=1_{B}$ ae $(m)$, where $\tilde{T}(\cdot)$ is an operator on $L_{\infty}(m)$ defined by

$$
\tilde{T}(f) \equiv(d / d m)\left[\left(f^{+} d m\right) T\right]-(d / d m)\left[\left(f^{-} d m\right) T\right],
$$

where $f$ in $L_{\infty}(m)$ is decomposed because $f d m$ need not be a signed measure. Such invariant sets are identical with those more typically defined by the requirement that $p(x, B)=1_{B}$ ae $(m)$, by $[3, \mathrm{p}$. 77]. The operator $\widetilde{T}$ will prove useful when characterizing the geometry of ergodic $m$.

From here on, $X$ is at least a $\sigma$-compact, locally compact Hausdorff space, $\mathscr{B}$ its Baire sets, and $m$ is a Baire measure. $(\mathscr{N}, \tau)$ denotes the collection of all Baire measures on $\mathscr{B}$ with the weak topology $\tau$ induced by the continuous functions with compact support, $C_{c}(X)$. A net $\left\{v_{\alpha}\right\}$ in $\mathscr{N}$ converges weakly to $m$ in $\mathscr{N}$ iff for all $f$ in $C_{c}(X): \int_{X} f d v_{\alpha} \rightarrow \int_{X}^{\alpha} f d m$. Note that $(\mathscr{N}, \tau)$ is a subspace of the locally convex topological vector space of linear, but not necessarily continuous, functionals on $C_{c}(X)$.

$X$ can be written as $\bigcup_{1}^{\infty} K_{n}$ and $\bigcup_{1}^{\infty} \mathcal{O}_{n}$, where the $K_{n}$ and $\mathcal{O}_{n}$ are, respectively, compact Baire sets and open bounded Baire sets such that $\mathcal{O}_{n} \subset K_{n} \subset \mathcal{O}_{n+1} \subset K_{n+1}$. Subsequently, there is a useful realization of $X$ as $\bigcup_{1}^{\infty} E_{n}$ where the $E_{n}$ are open bounded Baire sets identified as $E_{1}=\mathcal{O}_{1}$, $E_{2}=\mathcal{O}_{2}$, and for $n \geqq 3, E_{n}=\mathcal{O}_{n}-K_{n-2}$. Any element of $X$ lies in at most two of the $E_{n}$. Using only those $E_{n}$ for which $m\left(E_{n}\right)>0$, the function

$$
u(x) \equiv \sum_{n} 1_{E_{n}}(x) \cdot\left[2^{n} m\left(E_{n}\right)\right]^{-1}
$$

lies in $L_{1}(m) \cap L_{\infty}(m)$, and one can quickly show, for invariant $m$, that the conservativeness of $m$ can be written as

$$
\begin{aligned}
X & =C(m) \Leftrightarrow \text { for all } E_{n} \text { such that } m\left(E_{n}\right)>0: \sum_{1}^{\infty} p^{k}\left(x, E_{n}\right) \\
& =\infty \text { ae }(m) \text { on } E_{n} .
\end{aligned}
$$

In the following the notation $E_{n}$ will refer to this particular realization of $X$.

2. An integral representation. Let $\mathscr{C}$ denote the conservative invariant elements of $\mathscr{N}$. It is easily shown that $\mathscr{C}$ is a positive convex cone. By definition, a presubinvariant $m$ in $\mathscr{N}$ is ergodic iff $\Sigma(m)$ is trivial. The ray, $\rho(m)$, generated by nonzero $m$ in $\mathscr{C}$ is extreme by definition iff convex representations of any element of $\rho$ always involve only other elements of $\rho$.

2.1. Proposition. A nonzero element $v$ of $\mathscr{C}$ is ergodic $\Leftrightarrow \rho(v)$ is extreme. 
Proof. Let $\nu$ be ergodic with $\nu=a \mu_{1}+(1-a) \mu_{2}, a \in(0,1)$, and $\mu_{1}, \mu_{2} \in \mathscr{C}$. Then $\mu_{1} \ll v, \mu_{2} \ll v$, and $\mu_{1}$ and $\mu_{2}$ cannot be mutually singular so that $\mu_{i}=\lambda_{i}+w_{i}$, where $\lambda_{1} \perp \mu_{2}$ and $\lambda_{2} \perp \mu_{1}$ and $0 \neq w_{1} \ll \mu_{2}, 0 \neq w_{2} \ll \mu_{1}$.

Denoting $d \mu_{2} / d v$ by $f_{2}$, we have $0 \leqq f_{2}$ is $\mathscr{B}$-measurable and finite ae $(v)$ on bounded sets. We can decompose $X=\bigcup_{1}^{\infty} B_{n}$, the $B_{n}$ disjoint bounded Baire sets, so that the functions $g_{k}$ defined as $\left(f_{2} \wedge k\right) \cdot 1 \cup_{1}^{k} B_{n}$ satisfy $g_{k} \uparrow f_{2}$ on $X$ and $g_{k}$ is in $L_{1}(v) \cap L_{\infty}(v)$. The operator $(\cdot) T$ acting on $L_{1}(v)$ via $\left(g_{k}\right) T=(d / d v)\left[\left(g_{k} d v\right) T\right]$ is well extended, see [3, pp. 4-5], to act on $\mathscr{B}-$ measurable positive functions by $\left(f_{2}\right) T \equiv \lim _{k}\left(g_{k}\right) T$. It follows from the invariance of $v$ that $f_{2} T=f_{2}$ ae $(v)$, or since the $g_{k}$ are in $L_{\infty}(v)$ and since $\tilde{T}(\cdot)$ acting on $L_{\infty}(v)$ is precisely this extension of $(\cdot) T$, that $\tilde{T} f_{2}=f_{2}$ ae $(v)$. By [3, p. 76], $v$ is conservative with respect to the adjoint of $\tilde{T}$. So by [3, p. 21] $f_{2}$ is $\Sigma(v)$-measurable and there exists a constant $c_{2}$ such that $\mu_{2}=c_{2} v$. Similarly, $d \mu_{1} / d v=c_{1}$ ae $(v)$, and so $\mu_{1}$ and $\mu_{2}$ lie on $\rho(v)$. The converse is quickly shown by contradiction and the proposition is proven.

Two nonzero ergodic elements of $\mathscr{C}$ are either mutually singular or proportional. Referring to [6], if $\mathscr{C}$ were weakly closed in $\mathscr{N}$, we would have the notion of caps, $\mathscr{H}$, in $\mathscr{C}$ defined as $\mathscr{H} \equiv\{\lambda \in \mathscr{C}: M(\lambda) \leqq 1\}$, using a gauge functional $M$. The set $\mathscr{H}_{1} \equiv\{\lambda \in \mathscr{C}: M(\lambda)=1\}$ will be called the cap lid. From [5, p. 236], the extreme points, $\operatorname{Ex}(\mathscr{H})$, of a cap in $\mathscr{C}$ are precisely the vertex $\{0\}$ and the elements in the cap lid located on extreme rays of $\mathscr{C}$. So for any cap $\mathscr{H}$, the set $\mathscr{E}\left(\mathscr{H}_{1}\right) \equiv \operatorname{Ex}(\mathscr{H})-\{0\}$ is exactly representative of all nonzero singular ergodic measures in the cone $\mathscr{C}$.

2.2. THEOREM (INTEGRAL REPRESENTATION). If the transition function $p(x, B)$ satisfies conditions

(i) $\mathscr{C} \neq\{0\}$,

(ii) $T(\cdot): C_{c}(X) \rightarrow C_{c}(X)$, where $T f(x) \equiv \int f(y) p(x, d y)$,

(iii) for any open bounded Baire set $\mathcal{O}$ such that $\hat{\mathcal{O}}$ is unbounded, the set $\left\{x \in \mathcal{O}: \sum_{1}^{\infty} p^{n}(x, \mathcal{O})<\infty\right\}$ is open, then every nontrivial $m$ in $\mathscr{C}$ lies in some cap lid $\mathscr{H}_{\mathbf{1}_{m}}$ and there is a probability measure $p_{m}$ concentrated on $\mathscr{E}\left(\mathscr{H}_{\mathbf{1}_{m}}\right)$ so that for all $B$ in $\mathscr{B}: m(B)=\int_{\mathscr{E}\left(\mathscr{H}_{1_{m}}\right)} \lambda(B) d p_{m}(\lambda)$.

Proof. By a theorem of P. Meyer, [5, p. 238] and [6, p. 95], there is a cap $\mathscr{H}_{m}$ such that $m \in \mathscr{H}_{1_{m}}$ whenever $\mathscr{C}$ is weakly closed in $\mathscr{N}$. Under (ii), ( $\cdot) T$ acting on measures is an operator on $\mathscr{N}$. Given a net $\left\{v_{\alpha}\right\}$ in $\mathscr{C}, m=\tau$-limit $v_{\alpha}$ satisfies for all $f$ in $C_{c}(X): \int f d\left(v_{\alpha} T\right)=\int f d v_{\alpha}=\int T f d v_{\alpha} \rightarrow^{\alpha}$ $\int T f d m=\int f d(m T) \Rightarrow v_{\alpha} T=v_{\alpha} \rightarrow^{\alpha} m T \Rightarrow m T=m$. Thus weak limit points of $\mathscr{C}$ are invariant. An example to follow shows that $\mathscr{C}$ is generally not closed under (i) and (ii) in the sense that weak limit points may not be conservative. If $m\left(\hat{E}_{n}\right)<\infty, \hat{E}_{n} \in \Sigma(m) \Rightarrow \hat{E}_{n} \subset C(m)$. We therefore concern ourselves with those $E_{n}$ for which $m\left(\hat{E}_{n}\right)=\infty$, denoting a typical such $E_{n}$ 
in the remainder of this argument by $\mathcal{O}$ and the set $\left\{x \in \hat{\mathcal{O}}: \sum_{1}^{\infty} p^{n}(x, \mathcal{O})=\right.$ $\infty\}$ by $\hat{\mathcal{O}}_{\infty}$. By (iii), $\mathcal{O}-\hat{\mathcal{O}}_{\infty}$ is an open subset of $\mathcal{O}$ avoiding $\hat{\mathcal{O}}_{\infty}$. Suppose $m\left(\mathcal{O}-\hat{\mathcal{O}}_{\infty}\right)>0$. Then there is a compact Baire $K \subset \mathcal{O}-\hat{\mathcal{O}}_{\infty}, f \in C_{c}(X)$ with $0 \leqq f \leqq 1$ and $f=1$ on $K$ and 0 on $\left(\mathcal{O}-\hat{\mathcal{O}}_{\infty}\right)^{c}$ such that $0<m(K) \leqq \int f d m$, while for all $\alpha, \int f d v_{\alpha} \leqq v_{\alpha}\left(\mathcal{O}-\hat{\mathcal{O}}_{\infty}\right)=0$, a contradiction. Therefore, with the supplementary "recurrence" condition (iii), $m$ is conservative and $\mathscr{C}$ is weakly closed in $\mathscr{N}$. Applying the Choquet-Bishop-de Leeuw theorem as described by R. Phelps in [6, pp. 30-31], there is a probability measure $p_{m}$ on $\left(\mathscr{H}_{m}, \mathscr{S}\right)$, where $\mathscr{S}$ is the $\sigma$-algebra generated by the Baire sets of $\mathscr{H}_{m}$ and the set $\operatorname{Ex}\left(\mathscr{H}_{m}\right)$, so that $p_{m}\left(\mathscr{E}\left(\mathscr{H}_{1_{m}}\right)\right)=1$ and $p_{m}$ represents $m$ in the sense that for any continuous affine function $\phi$ on $\mathscr{H}_{m}: \phi(m)=$ $\int_{\mathscr{H}_{m}} \phi(\lambda) d p_{m}(\lambda)$. Let $K$ be any compact Baire set. There is a sequence $\left\{g_{n}\right\}$ in $C_{c}(X)$ such that $g_{n} \downarrow 1_{K}$ and a corresponding sequence $\left\{\phi_{n}\right\}$ of continuous $\mathscr{S}$-measurable affine functions on $\mathscr{H}_{m}$ such that $\phi_{n}(m) \equiv$ $\int_{X} g_{n} d m=\int_{\mathscr{H}_{m}} d p_{m}(\lambda) \int_{X} g_{n} d \lambda$. For some $\hat{n}, \phi_{\hat{n}}(m)<\infty$ and so for each $\lambda$ and $q=0,1,2, \cdots, \phi_{\hat{n}+q}(\lambda) \rightarrow a \lambda(K)$, so that

$$
m(K)=\int_{\mathscr{B}\left(\mathscr{H}_{1_{m}}\right)} \lambda(K) d p_{m}(\lambda) .
$$

Referring to $[\mathbf{1}$, p. 5], the class of compact Baire sets in $\mathscr{B}$ is a $\pi$-system. It is easily shown that the collection $\{B \in \mathscr{B}: \lambda(B)$ is $\mathscr{S}$-measurable and $\left.m(B)=\int_{\mathscr{E}\left(\mathscr{H}_{1_{m}}\right.}, \lambda(B) d p_{m}(\lambda)\right\}$ is a $d$-system and so equal to $\mathscr{B}$, yielding the theorem.

A more motivated argument towards the conservativeness of $m$ is provided by defining the support of $m$ as in [9, p. 308]. Then under condition (ii), $\hat{\mathcal{O}}$ is open so that $\operatorname{supp}(m) \cap \hat{\mathcal{O}}$ and $\hat{\mathcal{O}}_{\infty}$ are "similar" in that both sets are nonvoid and unbounded, both necessarily intersect $m$-positive open Baire subsets of $\hat{\mathcal{O}}$, and under (iii): $\operatorname{supp}(m) \cap \mathcal{O} \subset \hat{\mathcal{O}}_{\infty} \cap$ $\mathcal{O} \Rightarrow m\left(\mathcal{O}-\hat{\mathcal{O}}_{\infty}\right)=0$.

We remark that (ii) implies that each individual set $\left\{x: p^{n}(x, \mathcal{O})>0\right\}$ is bounded, but does not in view of the following example imply that their union, or $\hat{\mathcal{O}}$ in particular, is bounded.

3. The necessity of the recurrence hypothesis. An example, suggested by M. Rosenblatt, shows given (i) and (ii) that a supplementary condition like (iii) is necessary. Let $X$ be the real line $R$ with the usual topology and let $Z$ denote the integers. A continuous function $h(y)$ on $R$ is defined as follows for $0<p<\frac{1}{2}<q<1$ and $p+q=1$ : for $y \leqq 0, h(y) \equiv q$; for $y \geqq 0$, $h(y)$ is defined in piecewise fashion on the unit interval containing integers $i$ and $i+1$ as $q$ for $y=i, i+1$, as $p$ for $i+1 /(i+2) \leqq y \leqq i+1-1 /(i+2)$, as $q(1-\lambda)+\lambda p$ for $y=i+\lambda /(i+2)$ or for $y=i+1-\lambda /(i+2)$ with $0<\lambda<1$. Graphically, in each positive integral interval, $h(y)$ is an inverted trapezoid. 
Then for $y$ in $R$ and $B$ in $\mathscr{B}, p(y, B) \equiv h(y) 1_{B}(y+1)+[1-h(y)] 1_{B}$ $(y-1)$ is a Markov transition function. For fixed $x$ in $[0,1)$, denote $S_{x} \equiv\{i+x, i \in Z\}$. The transition function $p(i+x, A)$ on $\left(S_{x}, \mathscr{B} \cap S_{x}\right)$ corresponds to a random walk on $S_{x}$, and for $x \in(0,1)$ a conservative invariant measure for the corresponding process in $(R, \mathscr{B})$ is given by

$$
\begin{gathered}
v_{x}(x-i)=(p / q)^{i-1}(1-h(x)) / q, \quad v_{x}(x)=1, \\
v_{x}(x+i)=[h(x) h(x+1) \cdots h(x+i-1)] \div[1-h(x+1)] \cdots \\
{[1-h(x+i-1)][1-h(x+i)] \text { for } i=1,2,3, \text { etc. }}
\end{gathered}
$$

So condition (i) is satisfied. As well, condition (ii) is fulfilled since for $f$ in $C_{c}(X)$,

$$
\int f(y) p(z, d y)=h(z) f(z+1)+[1-h(z)] f(z-1) .
$$

However, the net $\left\{v_{x}\right\}$ in $\mathscr{C}$ converges weakly as $x \rightarrow 0$ to the invariant measure $m=\left\{\left(q_{i}^{i} p\right)^{i}\right\}_{i \in Z}$ which is not conservative since for $p \neq q$ there are no recurrent states and so no conservative presubinvariant measures on $Z$. So $\mathscr{C}$ is not, in general, weakly closed under (i) and (ii) and for example need not have a compact base.

4. Finite measures. In [7, p. 100], Lemma 1 states that the space of regular Borel probability measures on a compact $X$ is weak-star compact. We could substitute Baire sets for Borel sets in the argument to obtain the weak-star compactness of the space $\mathscr{R}$ of Baire probabilities on $X$. Let $\mathscr{P}$ be the convex subspace of invariant (and so conservative) Baire probabilities. Condition (ii), which now reads $T: C(X) \rightarrow C(X)$, ensures that $\mathscr{P}$ is weakly closed in $\mathscr{R}$. $\mathscr{P}$ is subsequently weakly compact since the weak and weak-star topologies inherited by $\mathscr{R}$ have the same subbasis with sets of the form $\left\{v \in \mathscr{R}:\left|\int f d v-\int f d v_{0}\right|<\varepsilon\right\}$. Condition (ii) ensures $\mathscr{P} \neq\{0\}$, from [7, p. 101]. Another result in [8] says that $\operatorname{Ex}(\mathscr{P})$ consists precisely of ergodic elements of $\mathscr{P}$ since ergodicity there can be shown to be equivalent to ours. So with little effort we have:

4.1. Proposition. Let $X$ be compact. Then the condition $T(\cdot)$ : $C(X) \rightarrow C(X)$ suffices to ensure that the set of invariant Baire probabilities, $\mathscr{P}$, is nonvoid and weakly compact and that for any $\mu$ in $\mathscr{P}$ there exists a probability measure $q_{\mu}$ so that for all $B$ in $\mathscr{B}: \mu(B)=\int_{\mathscr{O}(\mathscr{P})} \lambda(B) d q_{\mu}(\lambda)$, where $\mathscr{E}(\mathscr{P})$ is the set of ergodic elements of $\mathscr{P}$.

5. Transformation invariance. The invariance of finite measures is typically defined in representation oriented papers with respect to one or more measurable, measure-preserving transformations on $X$. See [2] for a history of such efforts. 
In [6, Chapter 10], there is an application of the Choquet-et al. theorem for transformation-invariant probabilities which is convenient for comparison. Let $X$ be compact and $\mathscr{P}$ be the collection of Baire probabilities invariant with respect to $p(x, B)$. Form the cartesian product $Y \equiv X^{Z}$ and the associated product $\sigma$-algebra $\mathscr{A} \equiv \mathscr{B}^{Z}$ which in fact, $[5$, p. 23], is the $\sigma$-algebra of Baire sets of $Y$. Denote the shift transformation-invariant measures by $\mathscr{P}_{\pi}$. There is an embedding $\Gamma: \mathscr{P}_{\rightarrow} \mathscr{P}_{\pi}$ via $\mu \rightarrow P_{\mu}$ where $P_{\mu}$ is the Carathéodory extension of the measure induced on any finite $X^{F}, F \equiv\left\{j_{1}, \cdots, j_{f}\right\}$ by

$$
P_{\mu, F}\left\{y \in X^{F}: y_{j_{i}} \in B_{i}, j_{i} \in F\right\}=\operatorname{prob}\left\{x_{0} \in B_{1}, x_{1} \in B_{2}, \cdots, x_{f-1} \in B_{f}\right\} .
$$

Moreover, using prediction theory, as in [7, p. 97] it can be shown that $\Gamma$ preserves ergodicity. If $\Gamma$ were surjective, one would then quickly subsume existing representations for shift transformation-invariant finite measures. But this is not the case. The process $\left\{y_{j}\right\}$ associated with $P_{\mu}$ is Markovian and stationary. For a given $X$, one need only produce a non-Markovian stationary process to exhibit a shift-invariant $P$ which is not the image under $\Gamma$ of any $\mu$ in $\mathscr{P}$.

\section{REFERENCES}

1. R. M. Blumenthal and R. K. Getoor, Markov processes and potential theory, Pure and Appl. Math., vol. 29, Academic Press, New York, 1968. MR 41 \#9348.

2. R. H. Farrell, Representation of invariant measures, Illinois J. Math. 6 (1962), 447-467. MR 27 \#265.

3. S. R. Foguel, The ergodic theory of Markov processes, Van Nostrand Math. Studies, no. 21, Van Nostrand-Reinhold, New York, 1969. MR 41 \#6299.

4. — Remarks on conservative Markov processes, Israel J. Math. 6 (1968), 381-383. MR 39 \#4939.

5. P. A. Meyer, Probability and potentials, Blaisdell, Waltham, Mass., 1966. MR 34 \#5119.

6. R. R. Phelps, Lectures on Choquet's theorem, Van Nostrand, Princeton, N.J., 1966. MR 33 \#1690.

7. M. Rosenblatt, Markov processes. Structure and asymptotic behavior, SpringerVerlag, Berlin and New York, 1971.

8. - Transition probability operators, Proc. Fifth Berkeley Sympos. Math. Statist. and Probability (Berkeley, Calif., 1965/66), Vol. II: Contributions to Probability Theory, Part 2, Univ. California Press, Berkeley, Calif., 1967, pp. 473-483. MR 35 \#3742.

9. H. Royden, Real analysis, Macmillan, New York, 1968.

Arthur D. Little, Inc., Cambridge, Massachusetts 02140

Current address: 45-573 Loihi Street, Kaneohe, Hawaii 